\title{
Diagnostic agronomique des pratiques culturales paysannes dans les vergers caféiers de Côte d'Ivoire
}

\author{
Christophe Eponon ${ }^{1, *}$, Didier Snoeck ${ }^{2}$, Emmanuel Kassin $^{3}$, Jules Keli ${ }^{3}$ et Daouda Kone ${ }^{1}$ \\ ${ }^{1}$ Laboratoire d'agrophysiologie et de pathologies végétales, UFR biosciences, université Félix Houphouët-Boigny, 22 BP 582 , \\ Abidjan 22, Côte d'Ivoire \\ ${ }^{2}$ UR systèmes des pérennes, TA B-34/2, avenue Agropolis, 34398 Montpellier Cedex 5, France \\ ${ }^{3}$ CNRA, Centre national de recherche agronomique, 01 BP 1001, Abidjan 01, Côte d'Ivoire
}

\begin{abstract}
Résumé - En Côte d'Ivoire, la production de café a connu une baisse depuis les années 1980. Les économistes l'ont expliquée par la baisse du prix du café par rapport au cacao, qui a poussé les producteurs à négliger la caféiculture au profit de la cacaoculture puis de l'hévéaculture. Pour comprendre les mécanismes agronomiques de cette baisse et les pratiques agricoles des planteurs, une enquête sur la conduite des vergers caféiers a été menée dans les principales régions productrices. Cent cinquante-six parcelles ont été visitées et les données ont été collectées à deux niveaux : auprès des caféiculteurs et par observation dans les parcelles. Les résultats montrent que la superficie moyenne des plantations de caféiers est de l'ordre d'un hectare. Les deux tiers du verger sont constitués de matériel végétal non sélectionné. Ce verger est vieillissant et les densités de plantation moyennes sont inférieures à 1960 caféiers/ha, densité recommandée par la recherche. Plus de la moitié des vergers sont conduits sous ombrage composé principalement d'un mélange d'arbres forestiers et d'arbres fruitiers. L'entretien des plantations se limite en moyenne à deux désherbages manuels par an et la lutte contre les insectes à un seul traitement insecticide avant la récolte. Moins de $10 \%$ des caféiculteurs apportent des fertilisants. Les techniques de conduite des caféiers par la taille et le recépage ne sont pas mises en œuvre correctement. En conséquence, le verger est peu productif et le rendement moyen n'est que de $325 \mathrm{~kg} / \mathrm{ha} / \mathrm{an}$. Ces pratiques très extensives et les vergers vieillissants reflètent l'intérêt limité des agriculteurs pour la caféiculture.
\end{abstract}

Mots clés : diagnostic / pratiques paysannes / vergers caféiers / Côte d'Ivoire

Abstract - Agronomic diagnosis of farmers' cropping practices in coffee orchards in Ivory Coast. Coffee production in Ivory Coast is declining since 1980. Economists have explained this by the lowering price of coffee compared to cocoa, prompting producers to neglect coffee for cocoa and then rubber. To understand the agronomic mechanisms of this decline and coffee farmers cropping practices, a survey was conducted in the main producing regions. One hundred and fifty-six coffee plots were visited and data were collected at two levels: interviews with farmers and observations in coffee plots. The results showed that the average coffee plantation has an area of about one hectare. Two thirds of the orchard consist of unsorted plant material. The orchard is old and the average planting densities are lower than 1960 coffee trees/ha, the recommended density. More than one half of the coffee orchards have shade trees consisting of a mixture of primarily forest and fruit trees. The maintenance of coffee plantations is reduced to two manual weedings per year and phytosanitary treatments are limited to one single insecticide application just before harvest. Less than $10 \%$ of farmers provide fertilizers to the coffee trees. Pruning and rejuvenation systems are not properly implemented. Therefore, the orchard is not productive and the average yield is only $325 \mathrm{~kg} / \mathrm{ha} /$ year. These extensive practices and the aging orchards both reflect the limited interest of farmers for coffee growing.

Keywords: diagnosis / farming practices / coffee crop / the Ivory Coast

\footnotetext{
$\bar{*}$ Auteur de correspondance $:$ eponon.eboa@yahoo.fr
} 


\section{Introduction}

Depuis son Indépendance, la performance économique de la Côte d'Ivoire a été le fait des exportations du secteur agricole (Esso, 2009). Les recettes liées à l'exportation des matières premières ont évolué au rythme de la production agricole. Le caféier (Coffea canephora P. var. robusta), qui a été pendant de nombreuses années la principale culture de rente en Côte d'Ivoire, connaît une baisse de production depuis 1980, suite à la baisse continue des cours sur le marché international. La production de café, estimée à $250000 \mathrm{t}$ en 1990 se situe à moins de $120000 \mathrm{t}$ en 2015 (FAOSTAT, 2015). Cette baisse fait suite à la régression drastique des prix d'achat du café aux producteurs, qui ont commencé à délaisser la culture du caféier au profit du cacaoyer dès les années 1980 (Léonard, 1997; Léonard et Oswald, 1995; Ruf, 1981). Par la suite, d'autres cultures pérennes comme le palmier et l'hévéa sont venues accentuer la reconversion des caféières (Koua, 2007; Ruf et Schroth, 2013). Cette situation a engendré une diminution des revenus de l'État et des producteurs. Malgré tout, le café représentait encore 1,4\% des exportations ivoiriennes en 2014/15 (Ministère de l'Agriculture, 2015). Bien que la sélection et la promotion de nouveaux clones et hybrides plus productifs que le matériel tout-venant, aient été faites par les instituts de recherches nationaux depuis les années 1960 pour soutenir quantitativement et qualitativement la production (De La Vaissiere, 1982), la caféiculture actuelle reste marquée par une faible utilisation du matériel végétal sélectionné, qui était cohérente avec la mise en culture de nouvelles terres sur des fronts pionniers. Au cours des années 2010 , le cacao a continué à dépendre en grande partie des défrichements de forêts; on observe aussi des investissements par défrichements de jachères et de vieilles caféières dans les régions où les réserves forestières s'épuisent (Ruf, 2000; Ruf et Schroth, 2013). Pour les quelques investissements dans de nouvelles caféières, le mouvement de reconversion serait encore plus net (Konate et al., 2015). Dans ce contexte de changement de précédents culturaux, une intensification du système de production incluant l'application d'engrais et de produits phytosanitaires sur des plants sélectionnés à fort potentiel de production (2,5 à $3 \mathrm{t} / \mathrm{ha}$ de café marchand) pourrait avoir des implications sur le développement et la production durable du café et, dans un contexte plus large, sur tout le secteur agricole. Toutefois, les informations détaillées sur les pratiques culturales villageoises en caféiculture ne concernent que la zone de production d'Aboisso (Koua, 2007). Le présent article vise à étendre et actualiser l'analyse des pratiques culturales du café à l'échelle nationale, une approche qui devrait contribuer à identifier les composantes agronomiques nécessaires à une remontée de la production de café en Côte d'Ivoire et à sa durabilité. Une enquête a été effectuée en vue d'établir un état des lieux des vergers caféiers en Côte d'Ivoire par la description des pratiques culturales dans les caféières et la caractérisation des principales zones de production.

\section{Matériels et méthodes}

\subsection{Cadre de l'étude}

Le café est produit dans sept régions : Lacs, Comoé, GôhDjiboua, Lagunes, Montagnes, Sassandra-Marahoué et BasSassandra. Les enquêtes ont concerné 156 parcelles de caféiers
Tableau 1. Taille moyenne des vergers de caféiers dans les différentes localités de la zone caféière.

Table 1. Average size of coffee orchards in the different localities of coffee area.

\begin{tabular}{lll}
\hline Régions & Localités & Superficies moyennes (ha) \\
\hline Comoé & Abengourou & $0,76 \pm 0,13 \mathrm{bc}$ \\
& Aboisso & $1,45 \pm 0,26 \mathrm{abc}$ \\
Lagunes & Adzopé & $0,41 \pm 0,19 \mathrm{c}$ \\
Gôh-Djiboua & Agboville & $0,81 \pm 0,20 \mathrm{bc}$ \\
& Divo & $0,76 \pm 0,14 \mathrm{bc}$ \\
Lacs & Oumé & $0,58 \pm 0,24 \mathrm{bc}$ \\
& Dimbokro & $1,05 \pm 0,19 \mathrm{abc}$ \\
Sassandra-Marahoue & Yamoussoukro & $1,30 \pm 0,24 \mathrm{abc}$ \\
& Vavoua & $1,00 \pm 0,29 \mathrm{abc}$ \\
& Bouaflé & $0,50 \pm 0,34 \mathrm{bc}$ \\
Montagne & Daloa & $1,94 \pm 0,19 \mathrm{a}$ \\
& Danané & $1,21 \pm 0,22 \mathrm{abc}$ \\
& Duekoué & $1,00 \pm 0,29 \mathrm{abc}$ \\
& Guiglo & $1,29 \pm 0,24 \mathrm{abc}$ \\
Bas-Sassandra & Man & $1,10 \pm 0,26 \mathrm{abc}$ \\
& San-Pedro & $0,55 \pm 0,26 \mathrm{bc}$ \\
& Sassandra & $1,68 \pm 0,29 \mathrm{ab}$ \\
& Soubré & $0,86 \pm 0,10 \mathrm{bc}$ \\
\hline
\end{tabular}

en production prises au hasard le long des axes routiers de 18 localités réparties dans ces sept régions ( $c f$. Tab. 1).

Le climat est de type subéquatorial. Le régime pluviométrique est bimodal, avec des précipitations moyennes variant de 1100 à $2300 \mathrm{~mm}$ par an (Yao et al., 2013). Les températures moyennes annuelles sont comprises entre 24 et $32^{\circ} \mathrm{C}$ (Kassin et al., 2008). La durée d'insolation moyenne est supérieure à 1900 heures par an (Yao et al., 2013).

La majorité des sols de la zone caféière ivoirienne sont des ferralsols caractérisés par une somme des bases échangeables inférieure à $8 \mathrm{méq} / 100 \mathrm{~g}$ de sol, un taux de saturation du complexe adsorbant inférieur à $80 \%$ et un pH oscillant entre 4,5 et 6,5 (Perraud, 1971).

\section{2 Échantillonnage et réalisation de l'enquête}

Les paysans propriétaires des parcelles sélectionnées ont été identifiés pour être interviewés individuellement sur leur plantation par des étudiants. Les informations fournies ont été complétées par des observations directes sur les parcelles. L'enquête a duré quatre mois, de septembre à décembre 2014.

\subsection{Données collectées et analyses statistiques}

Les informations collectées ont concerné la superficie, l'origine du matériel végétal planté, la densité de plantation, le système de conduite des vergers, le désherbage, les traitements insecticides, la fertilisation, le précédent cultural, la présence d'arbres d'ombrage, l'âge et la production des caféiers.

Les données ont été dépouillées et codifiées à l'aide du tableur Ms Excel. La codification attribue des valeurs élevées à l'adoption des bonnes pratiques culturales (CNRA, 2005). L'analyse statistique descriptive a permis de décrire les séries 
Tableau 2. Caractéristiques des pratiques agronomiques en milieu paysan et moyennes au niveau national. Table 2. Characteristics of agronomic practices in peasant environments and their averages at national level.

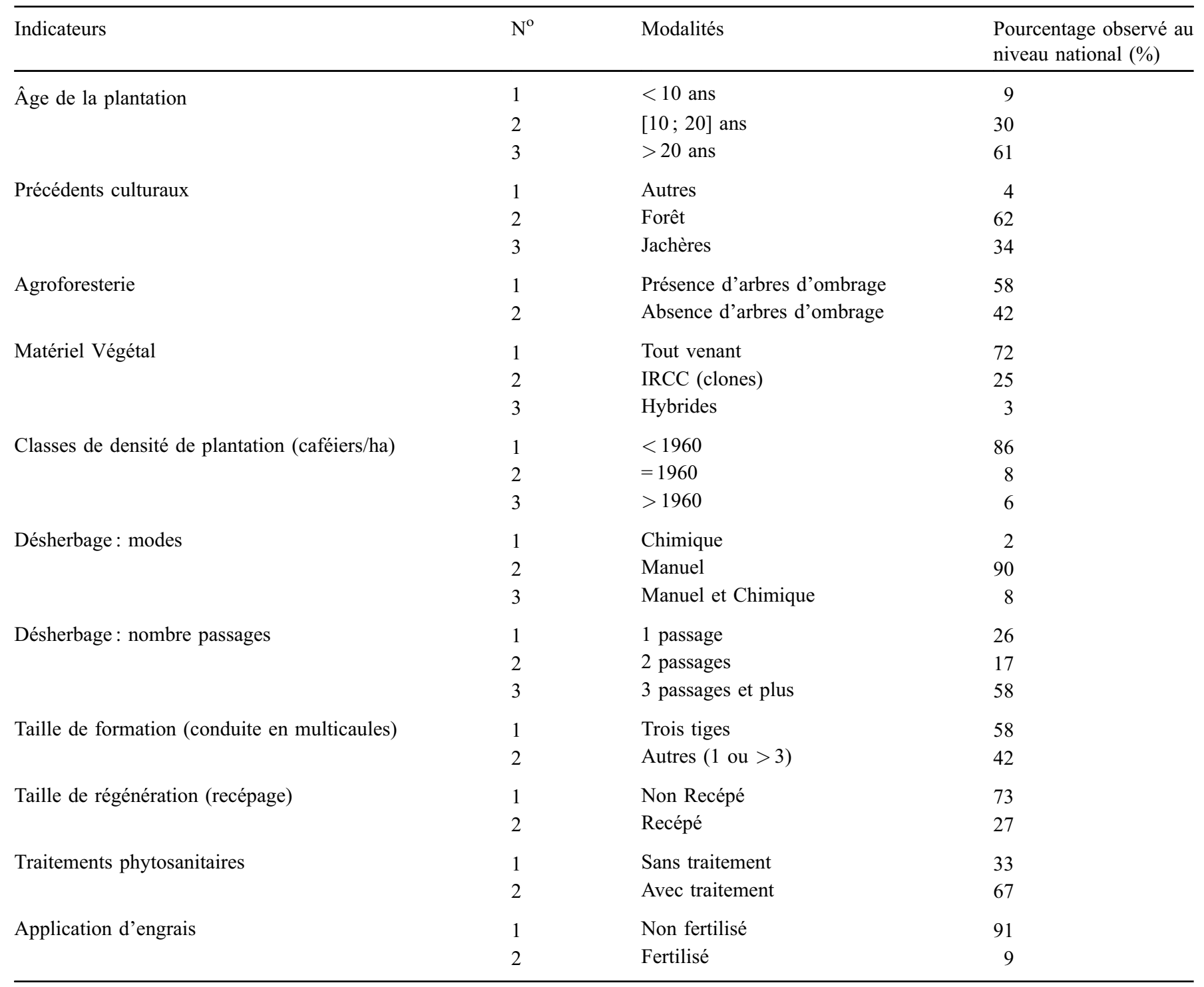

de valeurs. La méthode de comparaison des moyennes utilisée a été celle de Tukey, au seuil de probabilité de $5 \%$. Vu le nombre important de critères (variables) retenus pour qualifier un verger (individu), des analyses factorielles ont été utilisées. L'analyse en composante principales (ACP) a été effectuée afin d'analyser les données et de les visualiser sous forme de nuages de points dans des espaces géométriques. Ensuite, une classification ascendante hiérarchique $(\mathrm{CAH})$ a été réalisée pour regrouper les individus présentant des caractéristiques voisines.

\section{Résultats}

\subsection{Diagnostic des pratiques culturales.}

\subsubsection{Taille des parcelles de caféiers}

Les superficies des parcelles visitées varient en moyenne de 0,4 à 1,9 ha (Tab. 1).

L'analyse de variance a révélé des différences significatives $(F=3,51, p<0,001)$ entre les 18 départements pour la taille moyenne des plantations. Elles sont plus élevées à Daloa $(1,94 \pm 0,19$ ha), Sassandra $(1,68 \pm 0,29$ ha $)$, Aboisso $(1,45 \pm 0,26 \mathrm{ha})$ en moyenne et plus petites à Adzopé $(0,41 \pm 0,19$ ha $)$. La moyenne par caféiculteur pour l'ensemble des vergers enquêtés est de 0,96 ha.

\subsection{2 Âges des vergers}

La distribution des caféières en trois classes d'âges ( 0 à $10 \mathrm{ans}, 11$ à $20 \mathrm{ans}$ et plus de $20 \mathrm{ans}$ ), correspondant aux premier, deuxième et troisième cycles de culture, a montré que les plantations de plus de 20 ans représentent plus de $61 \%$ du verger caféier (Tab. 2). Tandis que $30 \%$ des plantations ont entre 11 et 20 ans et seulement $9 \%$ ont moins de 10 ans.

\subsubsection{Précédents culturaux des caféières}

Les caféiers ont été plantés après trois principaux types de précédents (Tab. 2). Le précédent « forêt» représente $62 \%$ du 


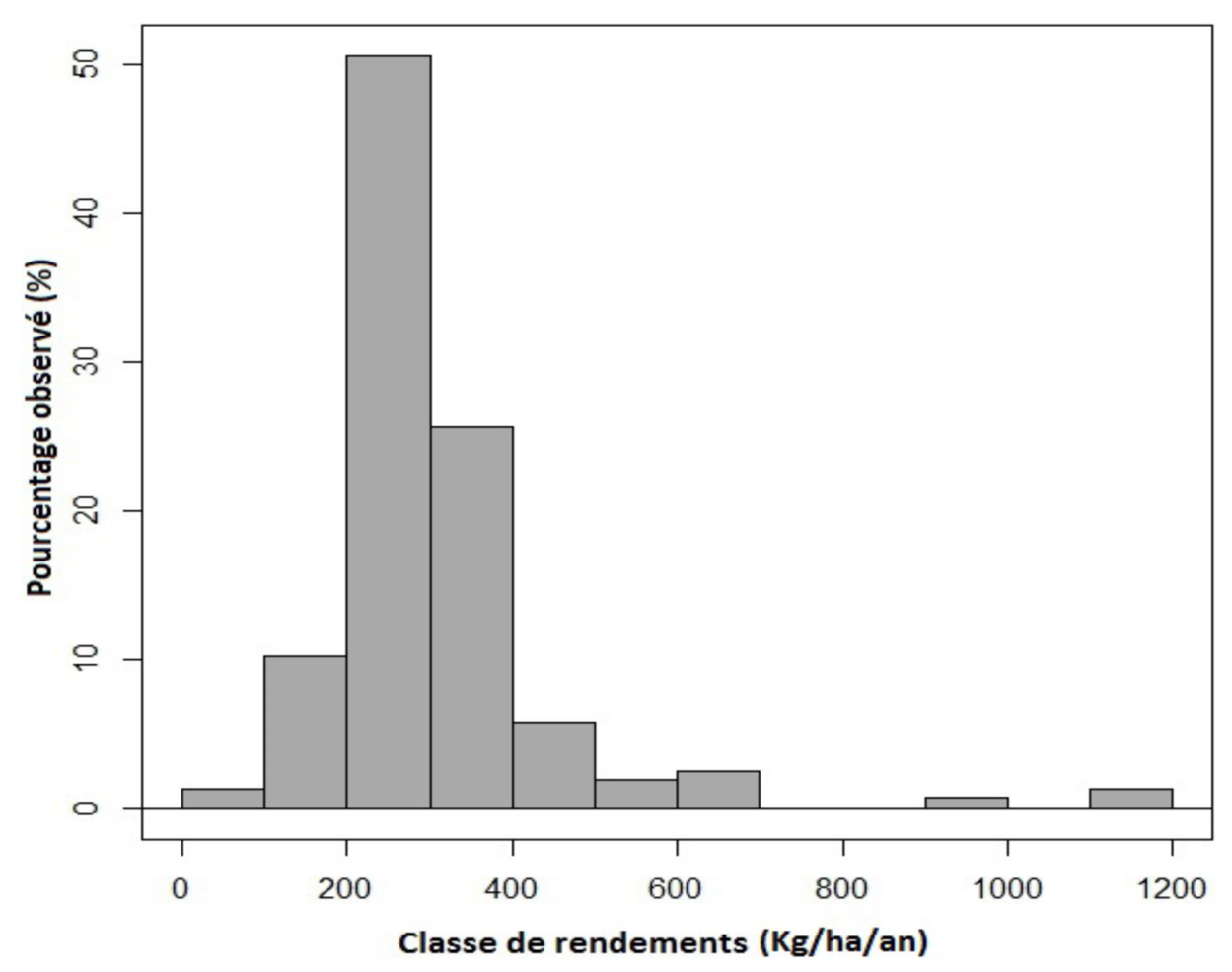

Fig. 1. Distribution des caféières par classes de rendements.

Fig. 1. Distribution of coffee by yield classes.

verger, contre $4 \%$ pour les jachères et $34 \%$ pour les anciennes plantations de caféiers.

\subsubsection{Matériel végétal}

Les plantations de caféiers ont été créées avec du matériel végétal d'origines diverses (Tab. 2). Le matériel végétal non sélectionné, prélevé par le caféiculteur dans sa parcelle et aussi appelé «tout venant», représente en moyenne $72 \%$ du verger. Seulement $28 \%$ des parcelles sont plantées avec du matériel végétal sélectionné : clones IRCC (25\%) ou nouveaux hybrides $(3 \%)$.

\subsubsection{Densité de plantation}

Seulement $8 \%$ des plantations ont la densité de 1960 caféiers/ha, considérée comme optimale par la recherche (Tab. 2). Tandis que $86 \%$ sont inférieures et $6 \%$ sont supérieures à la densité recommandée.

\subsubsection{Agroforesterie}

La culture du caféier sous ombrage est considérée comme un système agroforestier lorsque l'installation des vergers a été faite en conservant les arbres existant lors de la plantation. L'association d'arbres a été observée dans $58 \%$ des plantations tandis que la culture en plein soleil est réalisée dans $42 \%$ des cas (Tab. 2).

\subsubsection{Désherbage: mode et fréquences}

Les caféières sont désherbées de trois manières (Tab. 2): $90 \%$ à la machette, $2 \%$ à l'herbicide et $8 \%$ par des passages alternés de fauchage à la machette et d'utilisation d'herbicide.
La fréquence de trois passages et plus est adoptée dans $27 \%$ des vergers, la fréquence de deux passages dans $88 \%$ et celle d'un seul passage dans $17 \%$ des vergers.

\subsubsection{Types de tailles du caféier}

Taille de formation : $58 \%$ des plantations sont conduites sur deux ou trois tiges par pied (Tab. 2) selon les recommandations de la recherche. Les $42 \%$ restants sont conduits soit sur une seule tige, soit sur plus de trois tiges.

Taille de régénération: Le Tableau 2 montre que les caféières adultes non recépées représentent $73 \%$ des vergers. Seuls $27 \%$ des vergers ont été recépés au moins une fois.

\subsubsection{Traitements insecticides}

En moyenne, $33 \%$ des plantations ne sont pas traitées (Tab. 2). Celles qui le sont $(67 \%)$ reçoivent une seule application d'insecticide (Décis ou Cypercal) par an, juste avant la récolte des cerises pour éviter les morsures de fourmis.

\subsubsection{Fertilisation}

La plupart des sols sous caféiers (91\%) ne sont pas fertilisés (Tab. 2). Les engrais utilisés sont soit l'engrais cacao $\left(0 \% \mathrm{~N}-23 \% \mathrm{P}_{2} \mathrm{O}_{5}-19 \% \mathrm{~K}_{2} \mathrm{O}\right)$, soit l'urée (46-0-0), soit de la fumure organique (surtout les fientes de volailles).

\subsection{Productivité des caféiers}

Il n'existe aucune différence significative $(F=0,816$, $p>0,672)$ entre les localités pour les rendements moyens des vergers. La distribution de la production est uni-modale et légèrement dissymétrique (Fig. 1). 


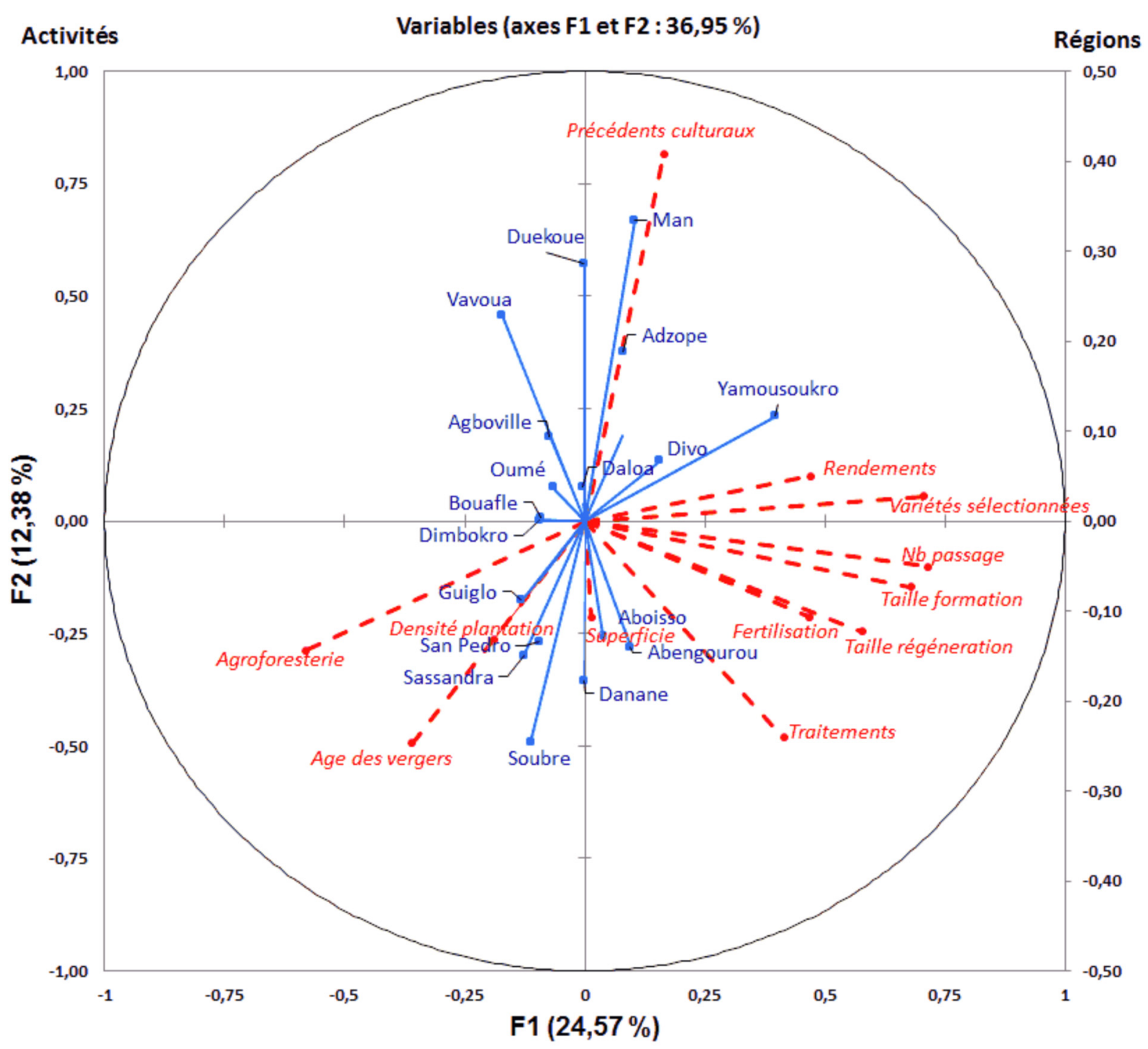

- Variables actives

- Variables supplémentaires

Fig. 2. Projection des variables et des individus sur le plan factoriel à deux dimensions (ACP).

Fig. 2. Projection of variables and individuals on the two-dimensional factor plane (PCA).

Les pourcentages de distribution les plus élevés ont été observés pour des valeurs comprises entre 200 et $400 \mathrm{~kg} / \mathrm{ha}$, soit $75 \%$ de la production. La moyenne pour l'ensemble du verger national est de $325 \mathrm{~kg} / \mathrm{ha}$ par an de café marchand.

\subsection{Caractéristiques des zones de cultures}

Une première analyse des zones a été réalisée en utilisant une analyse en composantes principales (Fig. 2).

Le nombre de composantes a été fixéà deux en se basant sur les critères de valeurs propres supérieures ou égales à un. La part d'information restituée par les deux composantes est de 51,8\% de la variance totale dont $31,6 \%$ pour l'axe 1 et $20,2 \%$ pour l'axe 2 .

L'axe F1 est lié aux "variétés sélectionnées》 et aux techniques d'entretien du côté positif. Il est lié aux variables «densité de plantation» et «agroforesterie» du côté négatif.
L'axe F2 est lié aux variables «rendements» et «précédents culturaux » du côté positif. Il est lié aux variables «âge des vergers» du côté négatif.

Le plan factoriel des individus permet également de représenter les localités sur le cercle à deux dimensions et ainsi d'identifier des tendances (Fig. 2).

Les positions des localités de Yamoussoukro et Divo du côté droit de l'axe F1, sont caractérisées par les variables, «nombre de passage », «taille de régénération», «taille de formation» et «variétés». Du côté gauche du même axe, les variables «agroforesterie» et «densité de plantation» caractérisent les localités de Vavoua, Bouaflé, Agboville, Dimbokro et Oumé.

$\mathrm{Au}$ niveau de l'axe F2 et du coté supérieur, les variables «précédents culturaux» et «rendements» caractérisent les localités de Man, Daloa Duekoué et Adzopé. À l'opposé du même axe, la variable «âge des vergers» caractérise les 
Tableau 3. Caractéristiques des régions en fonctions de quelques facteurs clés. Table 3. Characteristics of the regions according to a few key factors.

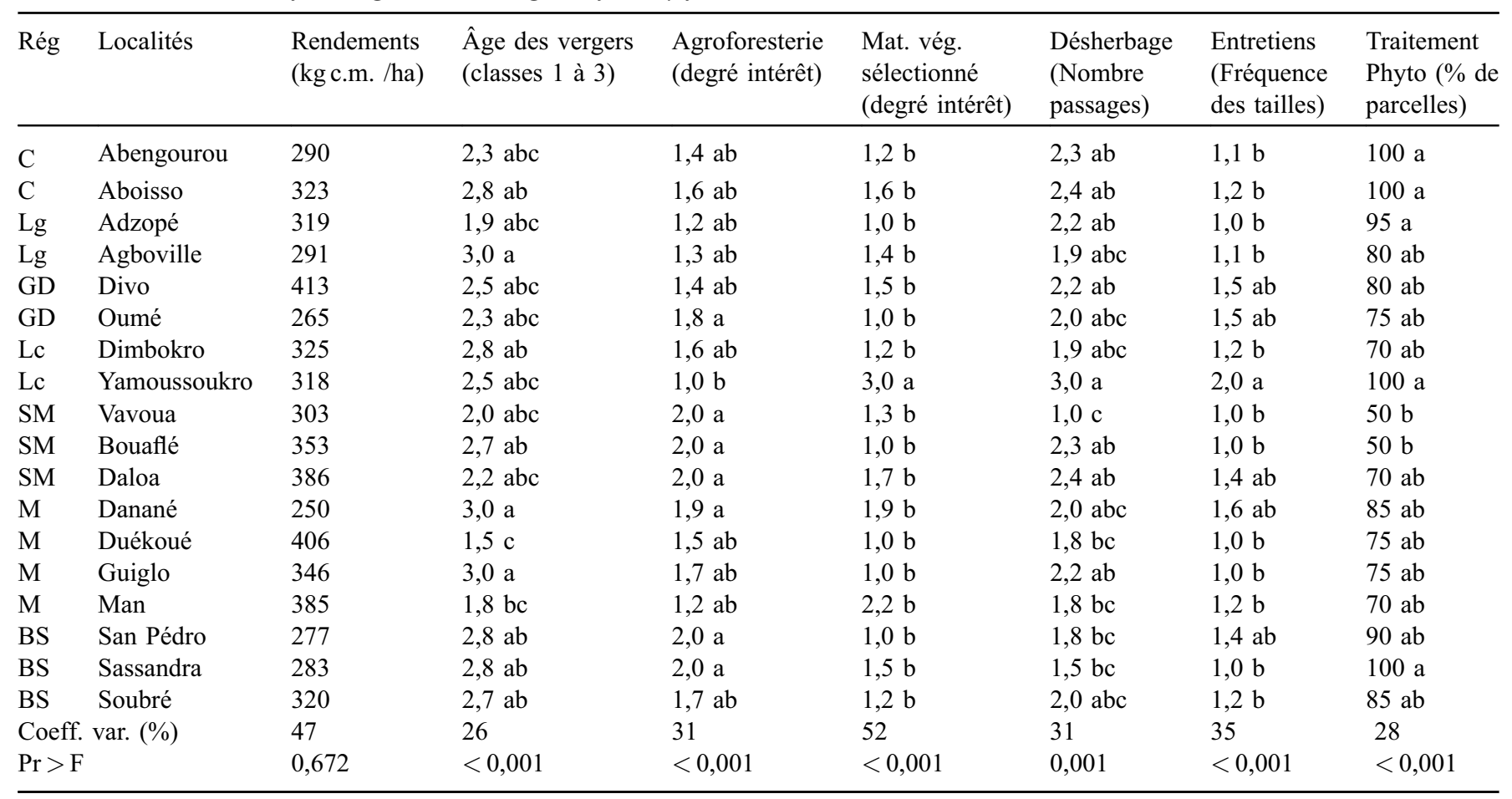

L: Lacs, C : Comoé, GD : Gôh-Djiboua, L : Lagunes, M : Montagnes, SM : Sassandra-Marahoue, BS : Bas-Sassandra, c.m. : café marchand, Rég: région, vég: végétal, Coeff. Var. : coefficient de variation.

localités de Danané, Aboisso, Abengourou, Sassandra, San Pedro, Soubré et Guiglo.

L'analyse de variance (Tab. 3) permet de confirmer les différences entre les localités en fonction des variables culturales étudiées.

Des ensembles plus homogènes et unifiés ont été obtenus par la classification hiérarchique. L'arbre hiérarchique a été construit avec la distance euclidienne et le critère de Ward (Fig. 3). Le regroupement hiérarchique a permis de distinguer quatre classes distinctes caractérisées par cinq variables. Ces variables qui caractérisent le mieux chaque partition (|Valeurtest $\mid \geq 2$ ), ont été consignées dans le Tableau 3. Ces variables ont été classées en fonction de leurs moyennes par comparaison avec des probabilités critiques inférieures à $5 \%$.

Le Tableau 3 et les Figures 2 et 3 montrent que:

- le groupe 1 comprend uniquement la localité de Yamoussoukro. Il est caractérisé par le recours à du matériel végétal sélectionné $(\mathrm{P}<0,001)$, à trois désherbages annuels $(\mathrm{P}<0,002)$ et à des densités de plantation non optimales $(\mathrm{P}<0,001)$;

- le groupe 2 regroupe les localités de Vavoua, Daloa, Duekoué et Man. Il est caractérisé par des exploitations sur des précédents culturaux non forestiers $(\mathrm{P}<0,001)$. Par contre, il rassemble de jeunes vergers $(\mathrm{P}<0,001)$ qui reçoivent de faibles applications de pesticides $(\mathrm{P}<0,02)$;

- le groupe 3 rassemble les localités de Divo, Adzopé, Oumé et Abengourou. Il est caractérisé par des exploitations de faibles surfaces $(\mathrm{P}<0,025)$ et des plantations de plein soleil $(\mathrm{P}<0,011)$;

- le groupe 4 concerne les localités de Bouaflé, Dimbokro, Guiglo, Agboville, San Pedro, Soubré, Danané, Sassandra et Aboisso. Il mêle des exploitations vieillissantes $(\mathrm{P}>0,003)$, qui ont été établies sur précédents forestiers $(\mathrm{P}>0,0008)$ avec maintien de l'ombrage $(\mathrm{P}>0,009)$.

\section{Discussion}

\subsection{Analyse des pratiques culturales}

Les recherches de Brou (2005) donnaient une superficie moyenne de plantation caféière par exploitation familiale encore assez importante, de l'ordre de 2 ha. En 2015, nos enquêtes ne recensent plus qu'un ha de café en moyenne. Cette baisse s'explique en partie par la stratégie de diversification des agriculteurs (Ruf et Schroth, 2013). Comme évoqué en introduction, plusieurs travaux de recherche montrent que de nombreuses vieilles caféières ont été reconverties en d'autres cultures arborées plus rentables comme le cacaoyer, le palmier à huile, ou l'hévéa. Les producteurs jugent le prix d'achat du café trop bas au regard des efforts à fournir dans les caféières. Selon Konate et al. (2015), environ $25 \%$ des vieilles caféières ont été reconverties en cacaoyères entre 2002 et 2003. L'analyse de l'âge des caféiers montre que le pourcentage des plantations qui ont un âge supérieur à 20 ans est passé de $43 \%$ (Brou, 2005) en 2005 à $61 \%$ en 2015. En 2015, les jeunes plantations ne représentent que $10 \%$ du verger, ce qui confirme le faible investissement dans la création de nouveaux vergers depuis une dizaine d'années (Koua, 2007). La remontée du prix d'achat du café aux planteurs de 166 FCFA à 423 FCFA entre les campagnes 2003/2004 et 2005/2006 n'a pas suffi à encourager les paysans à effectuer des investissements pour créer de nouvelles plantations de caféier. 


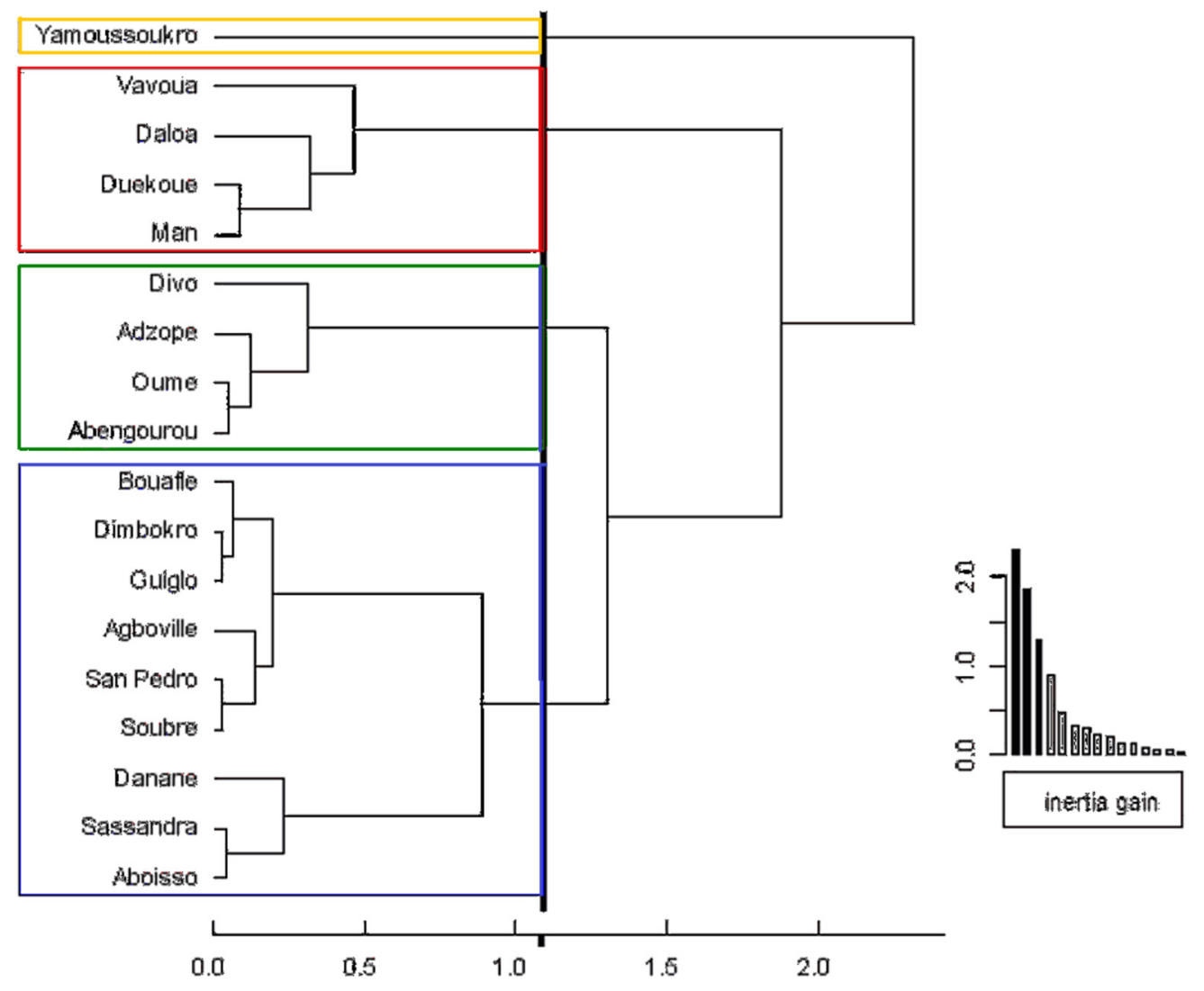

Fig. 3. Classification hiérarchique des 18 localités en fonction de leurs pratiques agricoles.

Fig. 3. Hierarchical classification of 18 localities according to their cropping practices.

En ce qui concerne le précédent cultural, l'extension des vergers de caféiers continue à se faire en majorité sur précédent forestier et les plantations restent dominées par l'utilisation de matériel végétal tout-venant, non sélectionné, en dépit des travaux de recherche de l'IFCC puis du CNRA et des efforts de vulgarisation par la SATMACI. Ce constat du faible impact de la création variétale et de sa diffusion par les centres de bouturage de la SATMACI, créés dans les zones de production, confirme les diagnostics antérieurs (Koua, 2007; Roux et Duris, 1995).

La présence d'arbres d'ombrage a été observée dans presque la moitié des exploitations de la zone de caféiculture. Les arbres sont constitués d'arbres forestiers épargnés lors de la mise en place du verger et d'arbres fruitiers (agrumes, manguier, colatier et avocatier). Les légumineuses, qui sont généralement recommandées pour leur capacité à fixer l'azote atmosphérique, ne sont que rarement observées. Pourtant, ces espèces peuvent apporter une source gratuite d'azote en plus d'un moyen de protection contre l'érosion, favorisant ainsi un système de culture durable avec faible utilisation d'intrants (Snoeck et al., 2000).

Les recommandations sur l'entretien des plantations ne sont non plus correctement appliquées. La densité optimale de 1960 arbres/ha, recommandée par Cestac et Snoeck (1982) n'est pas appliquée en milieu paysan. La fréquence de désherbages la plus citée par les agriculteurs est de deux fauchages par an, ce qui est bien inférieur aux cinq ou six recommandés (CNRA, 2005). Le premier passage est effectué quand les paysans jugent les adventices relativement gênantes et le second est opéré juste avant la récolte. Malgré le grand choix d'herbicides disponibles sur le marché, le désherbage chimique est très peu répandu. Les tailles de formation multicaule à trois tiges et le recépage périodique destiné à régénérer les caféiers, ne sont pratiquement pas exécutés. Les interventions phytosanitaires sont insuffisantes et inappropriées. Dans presque tous les cas, un seul traitement insecticide est réalisé, avant la récolte pour lutter contre les fourmis. Cette pratique n'est pas vraiment phytosanitaire, mais plutôt destinée à limiter les morsures douloureuses des cueilleurs de cerises. Pourtant une maladie et deux ravageurs (rouille des feuilles du caféier, scolyte du fruit du caféier et scolyte des rameaux du caféier) affectent considérablement la production. En particulier, les pertes provoquées par le scolyte des fruits peuvent varier, selon les estimations, de 0,6 à $11,6 \%$ voire plus (Wegbe et al., 1998). L'apport de fumure est une pratique presque inexistante en caféiculture. Les plantations fertilisées le sont avec des engrais non adaptés, tels que le 0-23-19 utilisé en cacaoculture qui n'apporte pas d'azote, alors que c'est l'élément le plus nécessaire aux caféiers.

Les résultats obtenus mettent en évidence les principales causes de la faible productivité des caféières ivoiriennes. Le rendement moyen de $327 \mathrm{~kg} / \mathrm{ha} / \mathrm{an}$ de café marchand est largement inférieur à la production de plus de $2000 \mathrm{~kg} / \mathrm{ha} / \mathrm{an}$ obtenue en station de recherche (N'Guetta et al., 2006), mais il est peu différent de la moyenne de production des autres pays africains, qui ne dépasse pas $400 \mathrm{~kg} / \mathrm{ha} / \mathrm{an}$ de café marchand 
(Jagoret et Descroix, 2002). Il apparaît donc que les travaux antérieurs de la recherche sur les «bonnes pratiques» de culture des caféiers ont eu un faible impact sur les pratiques paysannes. Mais il apparaît aussi que la rationalité est du côté des planteurs. Dans les conditions peu rémunératrices dans lesquelles ils se trouvent, les caféiculteurs n'ont probablement aucun intérêt économique à adopter des pratiques de culture intensives.

\subsection{Analyse des caractéristiques des zones de caféicultures}

Les localités ont été regroupées en quatre classes en fonction des pratiques agronomiques.

Dans la classe 1, la plupart des caféiculteurs ont eu recours au matériel végétal sélectionné pour la mise en place de leurs parcelles. La plantation de boutures sélectionnées issues des clones de l'IRCC avait été vivement encouragée par les structures de vulgarisation comme la SATMACI (De La Vaissiere, 1982). Les pratiques d'entretien relativement bonnes indiquent que les paysans se sont appropriés certaines pratiques qui perdurent malgré l'absence de structures d'encadrement efficaces. Les densités de plantation plus faibles par rapport à la moyenne générale traduiraient l'adoption de l'ancienne recommandation qui était de $1333 \mathrm{arbres} / \mathrm{ha}$, suivant l'écartement $3 \mathrm{~m} \times 2,5 \mathrm{~m}$ en rectangle conduits avec quatre tiges par pied (CNRA, 2005). Toutefois, les productions les plus élevées n'ont pas été obtenues dans cette région. En effet, l'utilisation de matériel végétal performant ne suffit pas à elle seule à obtenir des rendements élevés. Il faut également tenir compte de l'environnement pédoclimatique et de l'ensemble des techniques culturales. Parmi les données climatiques, la pluviométrie moyenne de $1100 \mathrm{~mm} / \mathrm{an}$ (Yao et al., 2013) est susceptible de limiter la production. En effet, les besoins en eau du caféier sont supérieurs à $1100 \mathrm{~mm} /$ an (Descroix et Snoeck, 2004).

Dans la classe 2, les exploitations sont caractérisées par des rendements relativement élevés par rapport à la moyenne générale. Les caféiers ont été mis en place sur précédents culturaux non forestiers. Il s'agit soit d'anciens champs reconvertis en caféières, soit de replantations sur de vieux vergers de caféiers. Les paysans appliquent peu d'insecticides. Les exploitations y sont relativement jeunes. Cet état de fait s'expliquerait par la vague de migration du front pionnier forestier au Centre-Ouest du pays pour la conquête de nouvelles terres cultivables (Freud et al., 2000) entraînant ainsi une main d'œuvre abondante et bon marché. Cette classe rassemble les localités de forte production. Toutefois, la forte production semble plus due au développement de la caféiculture grâce à la force de travail des migrants qu'à l'application de bonnes pratiques culturales.

Dans la classe 3, on observe les plus petites parcelles de caféiers ; ce qui s'expliquerait par la reconversion de certaines vieilles caféières en plantations d'autres cultures pérennes (palmier à huile, hévéa) suite à l'épuisement des réserves forestières (Ruf et Allangba, 2001). En matière de pratiques agricoles, les paysans pratiquent davantage la culture sans ombrage mais sans apport de fertilisants, ce qui ne contribue pas à assurer la productivité et la durabilité de la production. En effet, la culture en plein soleil permet d'accroître les productions, mais est plus exigeante en fertilisants. Or, ceux-ci ne sont pas apportés par les caféiculteurs.

Dans la classe 4, les caféiers ont été établis sur précédents forestiers. Le verger est vieillissant. Ce sont les anciennes zones de production de café du pays. On y trouve également des agriculteurs qui pratiquent la culture sous ombrage. Les caractéristiques de ce groupe tiennent au fait que la caféiculture a été introduite et installée en premier lieu dans ces zones forestières, avant de s'étendre à l'ensemble du pays. Le vieillissement du verger pourrait s'expliquer également par le processus de diversification encouragé par les politiques publiques et adopté par les paysans, notamment dans la région d'Aboisso dans les années 1980 pour la culture du palmier à huile (Ruf et Schroth, 2013).

\section{Conclusion}

Nos résultats contribuent à expliquer pourquoi la production caféière en Côte d'Ivoire est en baisse et pourquoi cette baisse risque de se prolonger. D'une part les planteurs optent pour des pratiques extensives, avec très peu d'intrants. Or ces systèmes extensifs atteignent leurs limites à cause de l'épuisement des réserves forestières. D'autre part, les planteurs investissent peu dans de nouvelles plantations de café. Ces stratégies paysannes semblent parfaitement rationnelles. Dans un contexte de prix bas et de diversification vers des cultures plus rémunératrices, les caféiculteurs ne voient pas d'intérêt, et n'ont probablement pas intérêt, à appliquer les «bonnes pratiques agricoles» recommandées par la recherche. Aujourd'hui, avec la replantation sur précédent cultural non forestier et dans un contexte de compétition avec d'autres cultures pérennes comme le cacao, l'hévéa et le palmier à huile, qui semblent plus profitables et qui bénéficient de dispositifs d'appui incitatifs, un regain d'intérêt des planteurs pour le caféier ne pourrait passer que par la mise en place de mesures incitatives de la part des acteurs de la filière café. Avec la rareté des facteurs de production (terre, main d'œuvre et capital d'exploitation), la redynamisation de la production du café doit certes se faire par l'intensification de la caféiculture en utilisant mieux les acquis de la recherche, mais aussi par une politique agricole s'intéressant plus à l'adoption des innovations par les petits producteurs. La question de prix d'achat du café plus incitatifs et plus rémunérateurs reste centrale. En conséquence, une évaluation complémentaire, dans les différentes régions, de la rentabilité économique des techniques intensives proposées serait nécessaire.

\section{Références}

Brou OL. 2005. Étude socio-économique du projet ochratoxine A. Rapport final, juillet 2005, 145 p. Disponible Sur http://www.fao. org/fileadmin/user_upload/agns/pdf/coffee/Annex-E.11.pdf.

Cestac Y, Snoeck J. 1982. Les essais de densités de dispositifs de plantation et de taille sur caféiers robusta en Côte d'ivoire. Résultats et perspectives. Paris: Café Cacao Thé, vol. XXVI, $n^{\circ} 3$, juil. Sept. 1982, pp. 183-198.

CNRA. 2005. Comment cultiver le caféier robusta en Côte d'Ivoire. Fiche technique. Centre national de recherche agronomique (CNRA), 5 p. Disponible sur http://www.erails.net/images/cotedivoire/cnra/cnra/file/ftech\%20caf\%C3\%A9.pdf. 
De La Vaissiere P. 1982. Évolution structurelle 1965-1975 de l'économie de plantation en Côte d'Ivoire: croissance sans changements techniques? Économie rurale, $\mathrm{n}^{\circ}$ 147-147, Janv. - Mars 1982, pp. 102-110. Disponible sur http://www.persee.fr/docAsPDF/ ecoru_0013-0559_1982_num_147_1_2849.pdf.

Descroix F, Snoeck J. 2004. Environmental factors suitable for coffee cultivation: In: Coffee: growing, processing, sustainable production. A guidebook for growers, processors, traders, and researchers. Weinheim: Wiley-CH, $1021 \mathrm{p}$.

Esso LJ. 2009. Dynamique des recettes du café et du cacao en côte d'ivoire. Cellule d'analyse de politiques économiques du CIRES (juillet 2009), 19 p. Disponible sur http://www.izf.net/sites/default/ files/bupedndeg10.pdf.

FAOSTAT. 2015. http://faostat3.fao.org/browse/Q/*/F.

Freud EH, Petithuguenin P, Richard J. 2000. Les champs de cacao : un défi de compétitivité Afrique-Asie. Paris (France): Karthala et CIRAD, 207 p. Disponible sur http://horizon.documentation.ird.fr/ exl-doc/pleins_textes/pleins_textes_7/b_fdi_03_05/010021334.pdf.

Jagoret P, Descroix F. 2002. Évolution de la culture de Coffea canephora en Afrique et problématique de développement. In: Recherche et caféiculture. CIRAD-CP-CAFE. Montpellier: CIRAD-CP, pp 44-59 (Plantations, recherche, développement, Mai 2002).

Kassin KE, Doffangui K, Kouamé B, Yoro G, Assa A. 2008. Variabilité pluviométrique et perspectives pour la replantation cacaoyère dans le Centre Ouest de la Côte d'Ivoire. Journal of Applied Biosciences 12: 633-641.

Konate Z, Assiri AA, Messoum FG, Sekou A, Camara M, YaoKouame A. 2015. Antécédents culturaux et identification de quelques pratiques paysannes en replantation cacaoyère en Côte d'Ivoire. Agronomie Africaine 27 (3): 301-314.

Koua AHG. 2007. Situation de la production de café en côte d'ivoire: cas du département d'Aboisso. Disponible sur http://www. memoireonline.com/10/07/663/m_situation-production-cafe-coted-ivoire-aboisso3.html.

Léonard E. 1997. Crise écologique, crise économique, crise d'un modèle d'exploitation agricole: ajustements et recomposition sociale sur les anciens fronts pionniers ivoiriens. In : Contamin B, Memel-Fotê H, eds. Le modèle ivoirien en question: crises, ajustements, recompositions. Paris: Orstom-Karthala, pp. 393-413. Disponible sur http://horizon.documentation.ird.fr/exl-doc/pleins_ textes/pleins_textes_7/b_fdi_03_03/010012775.pdf.

Léonard E, Oswald M. 1 - 995 . Cocoa smallholders facing a double structural adjustment in: Côte d'Ivoire: responses to a predicted crisis. In: Ruf F, Siswoputranto PS, eds. Cocoa Cycles: the economics of cocoa supply. Cambridge: Woodhead Publishing Limited, pp. 151-160.

Ministère de l'Agriculture. 2015. Les politiques agricoles à travers le monde: quelques exemples. Ministère de l'Agriculture, de
l'Agroalimentaire et de la Forêt, $10 \mathrm{p}$. Disponible sur http:// agriculture.gouv.fr/politiques-agricoles-fiches-pays.

N'Guetta ASP, Kouamanan KB, Yapo AA, Lidah YJ. 2006. Amélioration génétique de Coffea canephora pierre par hybridation interspécifique : étude de descendances de première génération de Coffea canephora Pierre x Coffea congensis Froenhner. Agronomie africaine 18(3): 201-212. Disponible sur https://www.ajol. info/index.php/aga/article/viewFile/1693/591.

Perraud A. 1971. Les sols. In : Le milieu naturel de la Côte-d'Ivoire. Paris: Mém. ORSTOM, 50, pp. 265-391. Disponible sur http:// horizon.documentation.ird.fr/exl-doc/pleins_textes/pleins_tex tes_6/Mem_cm/16368.pdf.

Roux G, Duris D. 1995. Étude de faisabilité de relance caféière en République de Côte d'Ivoire. Première phase : diagnostic, rapport préliminaire. Courbevoie (France): SOCA2, 143 p.

Ruf F. 1981. Le déterminisme des prix sur les systèmes de production en économie de plantation ivoirienne. Cahiers du CIRES 28-29: $35-52$.

RufF. 2000. Déterminants sociaux et économiques de la replantation. Oléagineux, Corps Gras, Lipides 7(2): 189-196. Disponible sur http://www.ocl-journal.org/articles/ocl/pdf/2000/02/ oc1200072p189.pdf.

Ruf F, Allangba K. 2001. Décisions de plantation et replantation cacaoyères. Le cas des migrants Baoulés à Oumé (Côte d'Ivoire). In : Assamoi RY, Burger K, Nicolas D, Ruf F, de Vernou P, eds. L'avenir des cultures pérennes. 5-9 novembre 2001. Yamoussoukro (Côte d'Ivoire): BNETD \& CIRAD.

RufF, Schroth G. 2013. Économie et écologie de la diversification des cultures pérennes tropicales. In: Cultures pérennes tropicales: enjeux économiques et écologiques de la diversification. Versailles: Éditions Quae, pp. 5-30. Disponible sur http://excerpts.numilog. com/books/9782759218547.pdf.

Snoeck D, Zapata F, Domenach A. 2000. Isotopic evidence of the transfer of nitrogen fixed by legumes to coffee trees. Biotechnology Agron. Soc. Environ. 4(2): 95-100. Disponible sur http://cataloguebibliotheques.cirad.fr/cgi-bin/koha/opac-detail.pl?biblionumber= 6293.

Wegbe K, Decazy B, Cilas C, Djiekpor EK. 1998. Le scolyte des fruits du caféier Hypothenemus hampei Ferr. (Coleoptera, Scolytidae) au Togo. Méthode d'estimation du niveau d'infestation, des pertes et du seuil de dégât économique. In: Dix-septième colloque scientifique international sur le café, Nairobi (Kenya), 20-25 juillet 1997. ASIC. Paris: ASIC, pp 639-645. Disponible sur http://asic-cafe.org/fr/system/files/17_084.pdf.

Yao NR, Oule AF, N'goran KD. 2013. Étude de vulnérabilité du secteur agricole face aux changements climatiques en côte d'ivoire. Rapport final, mai 2013, 105 p. Disponible sur http://www.ci.undp. org/content/dam/cote_divoire/docs/rapports/PNUD\%20RAP PORT\%20FINAL\%20DEFINITIF\%202013.pdf?download.

Citation de l'article : Eponon C, Snoeck D, Kassin E, Keli J, Kone D. 2017. Diagnostic agronomique des pratiques culturales paysannes dans les vergers caféiers de Côte d'Ivoire. Cah. Agric. 26: 45007. 